\title{
Efeitos da auriculoterapia na ansiedade de gestantes no pré-natal de baixo risco
}

Effects of auriculotherapy on anxiety of pregnant women receiving low-risk prenatal care Efectos de la auriculoterapia en la ansiedad de mujeres embarazadas en atención prenatal de bajo riesgo

\author{
Hércules Luz da Silva ${ }^{1}$ ic https://orcid,org/0000-0002-0295-5586 \\ Márcia Valéria de Souza Almeida ${ }^{1}$ io https://orcid.org/0000-0002-1318-7084 \\ Júlia da Silva Papi Diniz ${ }^{1}$ ic hitps://orcid.org/0000-0002-6678-8705 \\ Franciéle Marabotti Costa Leite ${ }^{1}$ io https:/orcid.org/0000-0002-6171-6972 \\ Maria Aparecida Vasconcelos Moura² io https://orcid. org/0000-0001-9085-6897 \\ Maria Edla de Oliveira Bringuente ${ }^{1}$ io hitps://orid.org/0000-0002-5151-5368 \\ Camila Brandão-Souza ${ }^{3}$ io https://orcid.org/0000-0002-5241-2061 \\ Maria Helena Costa Amorim ${ }^{3}$ io hitps://orcid.org/0000-0002-4252-7092
}

\section{Como citar:}

Silva HL, Almeida MV, Diniz JS, Leite FM, Moura MA, Bringuente ME, et al. Efeitos da auriculoterapia na ansiedade de gestantes no pré-natal de baixo risco. Acta Paul Enferm. 2020;33:eAPE20190016.

DOI

http://dx.doi.org/10.37689/actaape/2020A00016

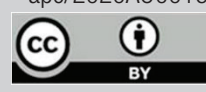

Descritores

Gravidez; Cuidado pré-natal; Ansiedade; Auriculoterapia; Terapias complementares

Keywords Pregnancy; Prenatal care; Anxiety; Auriculotherapy; Complementary therapies

Descriptores

Embarazo; Atención prenatal; Ansiedad; Auriculoterpia; Terapias complementarias

\section{Submetido \\ 25 de Janeiro de 2019 \\ Aceito \\ 29 de Outubro de 2019}

Autor correspondente

Camila Brandão-Souza milaunifesp@gmail.com

\section{Resumo}

Objetivo: Avaliar os efeitos da auriculoterapia nos níveis de ansiedade em gestantes atendidas em pré-natal de baixo risco.

Métodos: Trata-se de estudo clínico randomizado, simples-cego, realizado em um ambulatório de pré-natal de baixo risco em uma maternidade filantrópica, no Espírito Santo, Brasil, com amostra de 50 gestantes (25 em grupo controle e 25 em grupo experimental). Utilizou-se como intervenção a auriculoterapia da Medicina Tradicional Chinesa, que é uma das práticas utilizadas como acupuntura de microssistema. Para o tratamento com auriculoterapia, utilizou-se pontos do pavilhão auricular sendo respectivamente: Triângulo Cibernético (Shen Men, Rim e Simpático), que são caracterizados como pontos de abertura, usados para ativar os pontos a serem estimulados: Tronco Cerebral, Baço, Ansiedade. 0 conjunto desses pontos tem a função de acalmar a mente e 0 espírito, reduzir a ansiedade, bem como produzir analgesia e sedação. 0 tipo de semente utilizada na auriculoterapia foi mostarda amarela-colza. A intervenção proposta foi realizada pelo pesquisador, enfermeiro obstetra, especialista em acupuntura. Gestantes de ambos grupos, controle e intervenção, participaram de quatro consultas de enfermagem em pré-natal, porém somente 0 grupo intervenção foi submetido a três sessões de auriculoterapia nessas consultas, com intervalo de três dias entre cada sessão, totalizando 13 dias de acompanhamento. A resposta esperada foi a presença de sensibilidade nos locais dos pontos a serem aplicados; e a estimulação das sementes de colza, adesivadas com fita cirúrgica microporosa e hipoalergênica, foram realizadas pela manipulação manual da gestante. Levantamento através de instrumentos como formulários contendo as variáveis sociodemográficas e clínicas, e o Inventário de Traço e Estado de Ansiedade (IDATE) aplicado por enfermeira especialista em acupuntura, não tendo participação na intervenção de Enfermagemauriculoterapia, para se evitar o viés do estudo. Realizou-se a análise descritiva dos dados, através de tabelas de frequência com número e percentual para cada um dos itens dos instrumentos de pesquisa e utilizou-se gráfico box-plot para ilustração e melhor visualização dos resultados. 0 ANOVA foi utilizado para comparação dos diferentes momentos, e o t Student para comparação entre os grupos caso e controle. 0 grau de significância adotado foi de 5\%. 0 pacote estatístico SPSS 20 foi utilizado para análise.

Resultados: No primeiro momento ambos os grupos apresentaram Traço de Ansiedade e Estado de Ansiedade em nível médio, não houve diferença estatística entre os grupos, 0 que evidenciou homogeneidade da amostra ( $p=0,385$ e $p=0,352$, respectivamente); após a intervenção com auriculoterapia houve uma redução significante do Estado de Ansiedade do grupo intervenção $(p=0,033)$ entre a terceira e quarta consulta, 0 mesmo não ocorreu no grupo controle $(0,052)$.

Conclusão: A auriculoterapia pode ajudar a diminuir a ansiedade em gestantes durante o pré-natal de baixo risco, sendo uma prática integrativa e complementar em potencial no âmbito do Sistema Único de Saúde, tendo o enfermeiro acupunturista relevante importância nesse processo.

\section{'Universidade Federal do Espírito Santo, Vitória, ES, Brasil.}

2Escola de Enfermagem Anna Nery, Universidade Federal do Rio de Janeiro, Rio de Janeiro, RJ, Brasil.

'Escola Paulista de Enfermagem, Universidade Federal de São Paulo, São Paulo, SP, Brasil.

Conflitos de interesse: nada a declarar. 


\begin{abstract}
Objective: To evaluate the effects of auriculotherapy on anxiety levels of pregnant women receiving low-risk prenatal care.

Methods: This was a randomized, single-blind clinical study conducted at a low-risk prenatal clinic in a philanthropic maternity hospital in the state of Espírito Santo, Brazil, with a sample of 50 pregnant women (25 in control group; 25 in experimental group). The intervention used was Auriculotherapy of Traditional Chinese Medicine (TCM), which is one of the practices used as microsystem acupuncture. For auriculotherapy treatment, three ear points were used together, namely the Shen Men, Kidney and Sympathetic (in Portuguese they are called triângulo cibernético or "cyber triangle"). These are opening points used for activation of the following points to be stimulated: Brainstem, Spleen, Anxiety. All these points have the function of calming the mind and spirit, reducing anxiety and producing analgesia and sedation. The mustard seed (yellow rapeseed) was used for auriculotherapy. The proposed intervention was performed by the researcher, an obstetric nurse and acupuncture specialist. Pregnant women from both control and intervention groups attended four prenatal nursing consultations, but only the intervention group underwent three auriculotherapy sessions at these consultations, with a three-day interval between each session, totaling 13 days of follow-up. The expected response was the presence of sensitivity at the sites of points to be applied and stimulation of the point through the mustard seed attached with microporous, hypoallergenic surgical tape, which were performed by manual manipulation of pregnant women. The survey was performed through instruments such as forms containing sociodemographic and clinical variables, and the State-Trait Anxiety Inventory (STAl) applied by an acupuncture nurse who did not participate in the auriculotherapy-nursing intervention to avoid bias. Descriptive data analysis was performed through frequency tables with number and percentage for each item of the research instruments. A box-plot graph was used for illustration and better visualization of results. The ANOVA was used for comparison of the different moments, and the Student's T for comparison between the case and control groups. The significance level adopted was 5\%. The SPSS 20 statistical package was used for analysis.
\end{abstract}

Results: In the first moment, both groups presented medium level of Trait Anxiety and State Anxiety, and there was no statistical difference between groups, thereby demonstrating the sample homogeneity ( $p=0.385$ and $p=0.352)$. After the auriculotherapy intervention, between the third and fourth consultations, there was a significant reduction of State Anxiety in the intervention group ( $p=0.033$ ), but the same did not occur in the control group ( 0.052$)$.

Conclusion: Auriculotherapy is a potential integrative and complementary practice within the National Health System (Brazilian SUS) that can help reduce pregnant women's anxiety during low-risk prenatal care. The acupuncturist nurse has relevant importance in this process.

\title{
Resumen
}

Objetivo: Analizar los efectos de la auriculoterapia en los niveles de ansiedad de mujeres embarazadas en la atención prenatal de bajo riesgo.

Métodos: Se trata de un ensayo clínico aleatorizado, simple ciego, realizado en consultorios externos de atención prenatal de bajo riesgo en una maternidad filantrópica, en el estado de Espírito Santo, Brasil, con una muestra de 50 mujeres embarazadas (25 en grupo de control y 25 en grupo experimental). Se utilizó como intervención la auriculoterapia de la Medicina Tradicional China, que es una de las prácticas utilizadas como acupuntura del microsistema. Para el tratamiento con auriculoterapia, se utilizaron los siguientes puntos del pabellón auricular: Triángulo Cibernético (shenmen, riñón y sistema autónomo simpático), que se caracterizan como puntos de apertura, utilizados para activar los puntos que serán estimulados posteriormente: tronco cerebral, bazo y ansiedad. El conjunto de estos puntos tiene la función de calmar la mente y el espíritu, reducir la ansiedad, así como también producir analgesia y sedación. El tipo de semilla utilizado en la auriculoterapia fue mostaza amarilla o colza. La intervención propuesta fue realizada por el investigador, enfermero obstetra, especialista en acupuntura. Las mujeres embarazadas de ambos grupos, el de control y el experimental, asistieron a cuatro consultas de enfermería en atención prenatal, pero solo el grupo experimental recibió tres sesiones de auriculoterapia en esas consultas, con un intervalo de tres días entre cada sesión y un total de 13 días de seguimiento. La respuesta esperada fue la presencia de sensibilidad en los lugares de los puntos que serían aplicados. La estimulación de las semillas de colza, pegadas con cinta quirúrgica microporosa e hipoalergénica, fue realizada mediante la manipulación manual de la mujer embarazada. La recopilación se realizó a través de instrumentos como formularios con variables sociodemográficas y clínicas y el Cuestionario de Ansiedad Estado Rasgo (IDATE) aplicado por enfermera especialista en acupuntura, sin participación en la intervención de enfermería-auriculoterapia, para evitar el sesgo del estudio. Se realizó un análisis descriptivo de los datos mediante tablas de frecuencia con número y porcentaje en cada ítem de los instrumentos de investigación y se utilizó un gráfico de caja y bigote para ilustrar y visualizar los resultados. Para comparar los diferentes momentos, se utilizó el ANOVA, y el t de Student para comparar el grupo experimental y de control. El nivel de significación adoptado fue de 5\%. Se utilizó el paquete estadístico SPSS 20 para el análisis.

Resultados: En el primer momento, ambos grupos presentaron rasgo y estado de ansiedad de nivel medio, no hubo diferencia estadística entre los grupos, lo que demostró homogeneidad de la muestra ( $p=0,385$ y $p=0,352$ respectivamente). Luego de la intervención con auriculoterapia, hubo una reducción significativa del estado de ansiedad del grupo experimental $(p=0,333)$ entre la tercera y cuarta consulta. Esto no ocurrió en el grupo de control $(0,052)$.

Conclusión: La auriculoterapia puede ayudar a reducir la ansiedad de mujeres embarazadas durante la atención prenatal de bajo riesgo y es una práctica integradora y complementaria con potencial en el contexto del Sistema Único de Salud, proceso en el cual el enfermero acupunturista tiene una gran relevancia.

\section{Introdução}

A ansiedade é considerada uma "resposta fisiológica natural", causando os sintomas de taquicardia, sentimento de medo generalizado, medo de desastre eminente, tensão e inquietação. Esses sintomas também podem estar relacionados a doenças, como fobias, síndrome do pânico, transtornos obsessivo-compulsivos, ansiedade generalizada, entre outros. ${ }^{(1)}$

$\mathrm{Na}$ gestação, a ansiedade tornou-se um achado comum devido ao ciclo de vida vivenciado pela mulher, caracterizado como momento de fragilidade emocional, flutuação hormonal e mudanças sociais que influenciam diretamente em sua saúde emocional. ${ }^{(2)}$ 
Para compreender melhor as alteraçóes no período gestacional e as interações entre a função imune, o sistema endócrino e o estado psicológico muitos estudos têm se fundamento na psiconeroimunologia que evidencia as comunicaçóes bidirecionais entre os sistemas neuroendócrino, neurológico e imunológico. ${ }^{(3)}$

Estima-se que até $87 \%$ das gestantes busquem a Medicina Complementar e Alternativa (MCA) para tratarem de suas queixas, por serem formas de tratamento que náo usam medicamentos tradicionais. São muitas as terapias buscadas pelas gestantes, dentre elas, a massagem terapêutica, os suplementos vitamínicos, as plantas medicinais, as terapias de relaxamento e a aromaterapia. ${ }^{(4)}$

A Medicina Tradicional Chinesa (MTC) traz, no universo de suas práticas, a auriculoterapia, que utiliza o pavilhão auricular como local de intervençáo, pois possui a representatividade de todo o corpo humano. Nessa terapia busca-se tratar o desequilíbrio energético, que inclui a ansiedade em gestantes. ${ }^{(5)}$ Sua efetividade e efeito são representados pelo seu resultado instantâneo. ${ }^{(6)}$

A forma de aplicação pode ser feita desde o agulhamento em sessão, a pressão sob os pontos, as agulhas específicas em fita cirúrgica microporosa e hipoalergênica, as sangrias, as mini-ventosas, o moxabustão, até as sementes de mostarda em fita microporosa. Esta última modalidade é a mais apropriada por náo apresentar riscos de infecção local, por ser o pavilhão auricular muito vascularizado e em cartilagem na sua maior extensão. ${ }^{(7)}$

O Conselho Federal de Enfermagem, autarquia federal e órgão responsável por disciplinar o exercício da enfermagem no Brasil, estabeleceu e reconheceu, por meio da Resolução n ${ }^{\circ} 326 / 2008^{(8)}$ essa terapia como especialidade passível de execução pelo Enfermeiro, e que deve ser utilizada de forma complementar em suas açóes, sempre objetivando a promoção e a recuperaçáo da saúde e reabilitação na doença.

A intervenção de Enfermagem-auriculoterapia é uma prática integrante das Práticas Integrativas e Complementares (PICs) no contexto da MTC, classificada como um procedimento de baixo custo e de fácil aplicação. Sua prática favorece uma assistência humanizada à gestante já que o enfermeiro obstetra e acupunturista desempenha um importante papel na assistência pré-natal com a intervenção, utilizando um enfoque que promova a diminuição do estado de ansiedade e de estresse na gravidez. ${ }^{(9)}$

No contexto do Sistema Único de Saúde (SUS), o sistema de saúde pública do Brasil, ter a possibilidade de associar as especializaçóes acupuntura e a Enfermagem obstétrica ao profissional enfermeiro possibilitou a implementação deste estudo, objetivando uma assistência e cuidado centrado no período gestacional, oportunizando às gestantes a prática intervenção de Enfermagemaauriculoterapia, de modo a assegurar a integralidade da atençáo e a totalidade do ser humano em seus vários ciclos de vida, proporcionando seu equilíbrio mental -orgânico/estrutural-emocional, de forma humanizada e centrada no conhecimento científico.

Diante do exposto o presente estudo tem como objetivo avaliar os efeitos da auriculoterapia nos níveis de ansiedade em gestantes atendidas em prénatal de baixo risco.

\section{Métodos}

Trata-se de estudo clínico randomizado, simples-cego, realizado em um ambulatório de pré-natal de baixo risco em uma maternidade filantrópica, no Espírito Santo, Brasil. Foi realizado cálculo estatístico para determinar o tamanho da amostra, onde os parâmetros adotados foram: poder de $80,0 \%, \alpha=0,05$ e diferença entre os grupos (40,0\%). O cálculo resultou em uma amostra de 50 gestantes, sendo 25 no grupo controle e 25 no grupo intervenção. Os grupos foram constituídos aleatoriamente, mediante sorteio prévio por programa estatístico. Incluiu-se gestantes que apresentaram as seguintes características: moradoras da Grande Vitória; entre a faixa etária de 18 a 42 anos; em qualquer idade gestacional; cientes e em concordância com a pesquisa; e que assinaram o Termo de Consentimento Livre e Esclarecido. Excluiu-se gestantes: com histórico de gestação de alto risco; que apresentavam problemas dermatológicos; que já tivessem sido submetidas à auriculoterapia; que apresentavam transtornos psiquiátricos graves; ou usuárias de drogas ilícitas. Aplicou-se a técnica de entrevista com registro em formulário contendo as variáveis sociodemográficas e clínico-obstétricas. Para se avaliar o traço e esta- 
do de ansiedade utilizou-se o Inventário de Ansiedade Traço-Estado (IDATE) desenvolvido por Spielberger, Gorsuch e Lushene (1970) e traduzido e adaptado para o Brasil por Biaggio e Natalício (1979), ${ }^{(10)}$ aplicado por enfermeira especialista em acupuntura, não tendo participação na intervenção de Enfermagemauriculoterapia, para se evitar o viés do estudo.

Esse inventário (IDATE) traz uma escala que avalia a ansiedade enquanto estado (IDATE-E), ou seja, que reflete uma reação transitória, uma sensação de momento, que pode ser alterada; e outra que avalia a ansiedade enquanto traço (IDATE-T), que reflete um aspecto mais estável do indivíduo em lidar com a ansiedade ao longo da vida, uma característica pessoal, inerente ao ser. A frequência do traço de ansiedade foi analisada por quatro categorias: quase sempre (4), frequentemente (3), às vezes (2), quase nunca (1); enquanto no estado de ansiedade estáo disponíveis as opçóes: não (1); um pouco (2), bastante (3), totalmente (4). A pontuação desses itens varia entre 20 e 80 pontos, podendo indicar níveis de ansiedade baixo (20 a 40), médio (40 a 60) e alto (60 a 80).

Em ambos instrumentos existem afirmaçóes em que os escores para análise são invertidos de 1, 2, 3, 4 para 4, 3, 2, 1, sendo eles Traço de Ansiedade (IDATE-T) - itens 1, 6, 7, 10, 13, 16 e 19, e o Estado de Ansiedade (IDATE-E) - itens 1, 2, 5, 8, $10,11,15,16,19$ e $20 .^{(10)}$

Para o tratamento com auriculoterapia, utilizou-se pontos do pavilháo auricular sendo respectivamente: Triângulo Cibernético (Shen Men, Rim e Simpático), que são caracterizados como pontos de abertura, usados para ativar os pontos a serem estimulados: Tronco Cerebral, Baço, Ansiedade. O conjunto desses pontos tem a funçáo de acalmar a mente e o espírito, reduzir a ansiedade, bem como produzir analgesia e sedação. $\mathrm{O}$ tipo de semente utilizada na auriculoterapia foi mostarda amarela-colza. ${ }^{(11-13)}$ A intervenção proposta foi realizada pelo pesquisador, enfermeiro obstetra, especialista em acupuntura e eletroacupuntura, com 8 anos de experiência clínica na MTC. A resposta esperada foi a presença de sensibilidade nos locais dos pontos a serem aplicados; e a estimulação das sementes de colza, adesivadas com fita cirúrgica microporosa e hipoalergênica, foram realizadas pela manipulação manual da gestante.
Gestantes de ambos grupos, controle e intervenção, participaram de quatro consultas de enfermagem em pré-natal, porém somente o grupo intervenção foi submetido a três sessôes de auriculoterapia nessas consultas, com intervalo de três dias entre cada sessão, totalizando 13 dias de acompanhamento $\left(1^{\circ}\right.$ consulta de enfermagem em pré-natal com aplicação do IDATE $+1^{\circ}$ sessão de auriculoterapia no grupo intervenção; intervalo de 3 dias; $2^{\circ}$ consulta de enfermagem em prénatal $+2^{\circ}$ sessão de auriculoterapia no grupo intervenção; intervalo de 3 dias; $3^{\circ}$ consulta de enfermagem em pré-natal $+3^{\circ}$ sessão de auriculoterapia no grupo intervenção e avaliação com Instrumento Estado de Ansiedade; intervalo de 3 dias; $4^{\circ}$ consulta de enfermagem em pré-natal e avaliação com Instrumento Estado de Ansiedade). Ao final da primeira sessão foi entregue um folder explicativo sobre a técnica de compressão e o número de vezes que o pavilhão auricular deveria ser estimulado em casa pela gestante (10 vezes ao dia, por um período aproximado de 10 segundos, entretanto se o ponto estivesse incomodando, o mesmo náo deveria ser estimulado). A quarta consulta de enfermagem teve objetivo de avaliar o Estado de Ansiedade das gestantes de ambos os grupos.

Enfatiza-se que ambos os grupos foram submetidos ao mesmo procedimento no que diz respeito às consultas de enfermagem em pré-natal, bem como aplicação do instrumento IDATE, exceto a intervenção de Enfermagem-auriculoterapia. Ressalta-se que todas as mulheres do grupo controle que desejaram, receberam o tratamento em questão após o desenvolvimento do estudo.

Para evitar o efeito Hawthorne, ou seja, para que não houvesse contaminação dos sujeitos do grupo controle com o grupo intervenção, as gestantes foram atendidas em dias diferentes da semana, de modo que elas não se encontrassem.

A coleta das informaçóes e o registro dos instrumentos foram iniciados somente após a aprovação do Comitê de Ética em Pesquisa do Centro de Saúde da Universidade Federal do Espírito Santo/ UFES, com número de Parecer 1.544.310, tendo o início em 15 de maio e o término 10 de outubro do ano de 2016.

Utilizou-se o programa Microsoft Office Excell 2010 for Windows para o registro e organização dos 
dados. Realizou-se análise descritiva através de tabelas de frequência com número e percentual; utilizou-se gráfico box-plot para ilustração e melhor visualização dos resultados. O ANOVA foi utilizado para comparação dos diferentes momentos, e o t Student para comparação entre os grupos caso e controle. $\mathrm{O}$ grau de significância adotado foi de $5 \%$. O pacote estatístico IBM SPSS 20 foi utilizado para análise.

\section{Resultados}

A tabela 1 apresenta a caracterização da amostra segundo variáveis sociodemográficas. A faixa etária mais frequente das gestantes foi de 18 a 24 anos

Tabela 1. Variáveis sociodemográficos das gestantes

\begin{tabular}{|c|c|}
\hline Característica & $\mathrm{n}(\%)$ \\
\hline \multicolumn{2}{|l|}{ Faixa etária } \\
\hline $18-24$ anos & $18(36)$ \\
\hline $25-29$ anos & 13(26) \\
\hline $30-34$ anos & 10(20) \\
\hline $35-39$ anos & $9(18)$ \\
\hline \multicolumn{2}{|l|}{ Estado civil } \\
\hline Solteira & $13(26)$ \\
\hline Casado/União estável & $33(66)$ \\
\hline Viúva & $4(8)$ \\
\hline \multicolumn{2}{|l|}{ Anos de estudo } \\
\hline Até 8 anos & $11(22)$ \\
\hline 9 anos ou mais & $39(78)$ \\
\hline \multicolumn{2}{|l|}{ Renda familiar } \\
\hline Até 2 salários & $38(76)$ \\
\hline 2-4 salários & $10(20)$ \\
\hline Mais 4 salários & $1(2)$ \\
\hline Não informou & 1(2) \\
\hline \multicolumn{2}{|l|}{ Ocupação } \\
\hline Empregada & $16(32)$ \\
\hline Desempregada & 19(38) \\
\hline Estudante & $3(6)$ \\
\hline Dona de casa & 10(20) \\
\hline Outros & $2(4)$ \\
\hline \multicolumn{2}{|l|}{ Crença } \\
\hline Católica & $15(30)$ \\
\hline Evangélica & $31(62)$ \\
\hline Espírita & $1(2)$ \\
\hline Mais de uma religião & 1(2) \\
\hline Nenhuma & $2(4)$ \\
\hline \multicolumn{2}{|l|}{ Suporte social } \\
\hline Família & $45(90)$ \\
\hline Amigos & $4(8)$ \\
\hline Ninguém & 1(2) \\
\hline Total & $50(100)$ \\
\hline
\end{tabular}

(36\%); juntamente com a categoria de 25 a 29 anos representaram $62 \%$ da amostra. Em sua maioria (66\%) eram casadas/união estável, com escolaridade $\geq 9$ anos de estudo (78\%), renda familiar de um a dois salários mínimos (76\%), desempregadas (38\%), evangélicas (62\%) e 90\% delas relataram possuir suporte familiar.

$\mathrm{Na}$ tabela 2 encontra-se dados relacionados ao perfil clínico-obstétrico das gestantes. Observa-se que 98\% tiveram a menarca antes dos 15 anos de idade, $56 \%$ a coitarca após essa mesma idade; $30 \%$ tiveram apenas uma gestação e $54 \%$ já haviam parido pelo

Tabela 2. Perfil clínico-obstétrico das gestantes

\begin{tabular}{|c|c|}
\hline Caracteristica & $n(\%)$ \\
\hline \multicolumn{2}{|l|}{ Idade da menarca } \\
\hline Até 15 anos & $49(98)$ \\
\hline Após 15 anos & $1(2)$ \\
\hline \multicolumn{2}{|l|}{ Idade coitarca } \\
\hline Até 15 anos & $22(44)$ \\
\hline Após 15 anos & $28(56)$ \\
\hline \multicolumn{2}{|l|}{ Número de gestações } \\
\hline Uma & $15(30)$ \\
\hline Duas & $16(32)$ \\
\hline Três & $10(20)$ \\
\hline Quatro ou mais & $9(18)$ \\
\hline \multicolumn{2}{|l|}{ Paridade } \\
\hline Uma & $27(54)$ \\
\hline Duas & 12(24) \\
\hline Três ou mais & $4(8)$ \\
\hline Nenhuma & $7(14)$ \\
\hline \multicolumn{2}{|l|}{ Filhos vivos } \\
\hline 1 filho & $34(68)$ \\
\hline 2 ou mais filhos & $16(32)$ \\
\hline \multicolumn{2}{|l|}{ Número de abortos } \\
\hline Nenhum & $43(86)$ \\
\hline Um aborto & $6(12)$ \\
\hline Dois ou mais abortos & 1(2) \\
\hline \multicolumn{2}{|l|}{ Tipo de parto } \\
\hline Normal & $19(38)$ \\
\hline Cesárea & $10(20)$ \\
\hline Ambos & $3(6)$ \\
\hline Nenhum & $18(36)$ \\
\hline \multicolumn{2}{|l|}{ Gravidez atual planejada } \\
\hline $\operatorname{Sim}$ & $23(46)$ \\
\hline Não & $27(54)$ \\
\hline \multicolumn{2}{|l|}{ Gravidez atual desejada } \\
\hline Sim & $46(92)$ \\
\hline Não & $4(8)$ \\
\hline \multicolumn{2}{|c|}{ Número de consultas de pré-natal } \\
\hline 1 consulta & $17(34)$ \\
\hline 2 a 5 consultas & $17(34)$ \\
\hline 6 ou mais consultas & $16(32)$ \\
\hline \multicolumn{2}{|l|}{ Número de internações } \\
\hline Nenhuma & $48(96)$ \\
\hline Uma ou mais & $2(4)$ \\
\hline Total & $50(100)$ \\
\hline
\end{tabular}


menos uma vez; 68\% tinham apenas um filho vivo. A maioria das gestantes não relatou abortos (86\%); $38 \%$ tiveram parto normal; mais da metade das mulheres não planejou a gravidez $(54 \%)$, todavia, a grande maioria desejou a gestação (96\%). Durante a gravidez, 96\% das gestantes não relataram internaçóes. A grande maioria não faziam uso de tabaco (96\%) e nem ingeria bebidas alcoólicas (94\%).

Observa-se na tabela 3 que o Traço de Ansiedade, que ambos os grupos, controle e intervenção, obtiveram o nível de média ansiedade. Não houve diferença estatística significante entre os grupos, evidenciando a homogeneidade $(p=0,385)$, bem como do Estado de Ansiedade na Primeira Consulta $(\mathrm{p}=0,352)$. Após intervenção com a auriculoterapia observa-se que, no grupo intervençáo, entre a terceira e a quarta consulta houve redução da ansiedade de forma significante $(\mathrm{p}=0,033)$, o mesmo não ocorreu no grupo controle $(0,052)$, o que reforça positivamente a efetividade dessa prática integrativa e complementar (Tabela 3/ Figura 1).

Tabela 3. Dados sobre ansiedade das gestantes

\begin{tabular}{lccc}
\hline Ansiedade & Controle & Experimental & $p$-value \\
\hline Traço & $50 \pm 11$ & $47 \pm 11$ & 0,385 \\
Estado & & & \\
$\quad$ Primeiro Momento & $46 \pm 13$ & $43 \pm 8$ & 0,352 \\
$\quad$ Terceiro Momento & $46 \pm 13$ & $41 \pm 8$ & \\
$\quad$ Quarto Momento & $44 \pm 12$ & $39 \pm 9$ & \\
$p$-value & 0,052 & 0,033 & \\
\hline
\end{tabular}

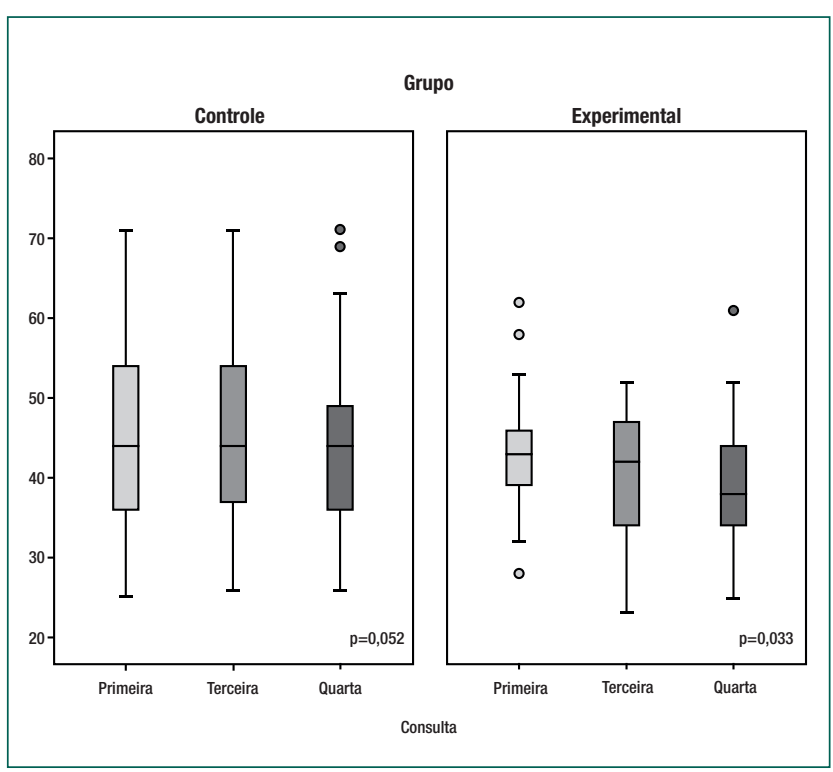

Figura 1. Nível de ansiedade das gestantes

\section{Discussão}

Este estudo mostrou que a auriculoterapia é um tratamento eficaz quando utilizado como intervenção visando a diminuição dos níveis de ansiedade nas gestantes atendidas em pré-natal de baixo risco.

A prevalência de transtornos mentais, tais como os de humor e ansiedade, afligem $20 \%$ das mulheres, ${ }^{(14)}$ os riscos obstétricos que envolvem o desenvolvimento de uma gestação colocam a mulher em estado susceptível à ansiedade, deixando-a dual: frágil, por medo das alteraçóes gravídicas, e ao mesmo tempo feliz pelo processo de concepção e formação de uma nova vida.

A ansiedade causa mudanças endócrinas e também comportamentais, como busca pelo tabaco, alimentação inadequada e não comprometimento com as consultas de pré-natal, ${ }^{(15)}$ e quanto maior o nível de ansiedade durante a gestação, maior será a probabilidade do surgimento de depressão puerpe$\mathrm{ral}^{(16)}$ e de efeitos deletérios, tanto para a gestante quanto para o recém-nascido. Em pesquisa longitudinal prospectiva, em que se avaliou a ansiedade em 35 mulheres grávidas, concluiu-se que esta tem influência na morfologia cerebral do feto e que este pode ter reduçóes regionais na massa cinzenta associada. Os autores relatam ser o estudo pioneiro e que demonstra que a ansiedade está relacionada a mudanças morfológicas no sistema nervoso central, levando a prejuízos intelectuais e cognitivos ao recém-nascido/criança. ${ }^{17)}$

Estudos epidemiológicos alertam para a subnotificação da ansiedade, seja por vergonha da mulher em se expressar, ou pela discriminação por considerar-se como sendo "apenas" um conjunto de sintomas passageiros, relacionados às mudanças hormonais. ${ }^{(18)}$ Pode ser amenizada quando há inclusão da família, amigos e parceiro(a) no processo gestacional. A gestante precisa de pessoas com as quais têm confiança para dividir medos, anseios e alegrias. ${ }^{(19)}$

A prematuridade e o baixo peso ao nascer de recém-nascidos de mães em estado de ansiedade vêm aumentando, quando relacionado a gestantes adolescentes e maiores de 30 anos. ${ }^{(20)}$ Devido a isso, há interesse em avançar em pesquisas para determinar essa associação, que prevalece em altas taxas, tanto em países em desenvolvimento como nos desenvolvidos. ${ }^{(21)}$ 
Estudo aponta que mulheres no puerpério, tanto parto normal quanto cesariana, apresentam nível médio de ansiedade. ${ }^{(22)}$ Outro estudo, que avaliou o estresse e depressão em puérperas na Inglaterra, sinaliza que mulheres com história de ansiedade no pré-natal apresentavam ansiedade no terceiro trimestre de gestação (13\%), já no pós-parto foram identificados apenas $8,1 \%$. Das Mulheres que apresentaram altos níveis de ansiedade no puerpério, 64\% relataram que a ansiedade estava presente no pré-natal. ${ }^{(23)}$

Um estudo experimental realizado no Brasil avaliou os efeitos do relaxamento na ansiedade em puérperas, concluiu que conhecer a gestante e seus sentimentos de forma geral proporciona melhor saúde mental no período gestacional, tendo como objetivo a redução de complicações no pós-parto, tanto para a mãe como para o recém-nato. ${ }^{(24)}$

As práticas integrativas estáo em constante avanço no meio das intervençóes em saúde, caracterizando-as em escolha de preferência para tratar transtornos de ansiedade, uma vez que possuem riscos diminuídos de efeitos adversos, bem como têm apresentado resultados positivos, demonstrando significativa redução da ansiedade e aumento no vínculo materno-fetal. ${ }^{(25)}$

De acordo o Ministério da Saúde, essas práticas devem ser implementadas para reduzir a ansiedade em gestantes, de modo que náo ocorram transtornos mais graves no pós-parto, sendo a auriculoterapia uma das Práticas Integrativas e Complementares em saúde que pode favorecer a redução da ansiedade. ${ }^{(26)}$

Este estudo apresentou como limitaçôes o número reduzido de gestantes de baixo risco no fluxo do ambulatório pesquisado.

\section{Conclusão}

Os resultados encontrados endossam a realização da auriculoterapia. A intervenção do profissional de saúde acupunturista em atendimentos de gestante na consulta pré-natal de baixo risco revelou-se significativo na comparação entre os grupos controle e intervenção com relação à diminuição da ansiedade, evidenciando essa prática da Medicina Tradicional Chinesa como prática favorável a ser aplicada no período gestacional na terapêutica do estado de ansie- dade. A prática da auriculoterapia pelo profissional enfermeiro foi bem aceita pelas gestantes durante a consulta pré-natal, bem como para o sistema de saúde, visto sua aplicabilidade e o baixo custo da terapêutica. Inúmeros foram os avanços das PICs no âmbito do SUS, a exemplo os tecnológicos, políticos e conceituais, porém muito esforço ainda é necessário para que essas intervençôes se tornem acessíveis à população. É uma realidade do SUS a carência de profissionais habilitados para as práticas da MTC, sendo esse um problema estrutural e organizacional da atenção básica em saúde.

\section{Agradecimentos}

À Pró-Matre; Ao Programa de Pós Graduação em Enfermagem-Mestrado Profissional da Universidade Federal do Espírito Santo.

\section{Colaborações}

Silva HL, Almeida MVS, Diniz JSP, Leite FMC, Moura MAV, Bringuente MEO, Brandão-Souza C e Amorim MHC contribuíram com a concepção do estudo, análise e interpretação dos dados, redação do artigo, revisão crítica relevante do conteúdo intelectual e aprovação da versão final a ser publicada.

\section{Referências}

1. Prado JM, Kurebayashi LF, da Silva MJ. Eficácia da auriculoterapia na redução de ansiedade em estudantes de enfermagem. Rev Esc Enferm USP. 2012;46(5):1200-6.

2. Araújo WS, Romero WG, Zandonade E, Amorim MH. Effects of relaxation on depression levels in women with high-risk pregnancies: a randomised clinical trial. Rev Lat Am Enfermagem. 2016;24(0):e2806.

3. Marques-DeakA, Sternberg E. Psiconeuroimunologia - a relação entre 0 sistema nervoso central e o sistema imunológico. Br J Psychiatry. 2004;26(3):143-4.

4. Hall HG, McKenna LG, Griffiths DL. Midwives' support for Complementary and Alternative Medicine: a literature review. Women Birth. 2012;25(1):4-12.

5. Maciocia G. Os fundamentos da medicina chinesa: um texto abrangente para acupunturistas e fitoterapeutas. 2a ed. São Paulo: Roca; 2007. 
6. Jimenez RN, Carvalho RA, Silvério-Lopes S, Suliano LC. Análise do Efeito Imediato da Auriculoterapia no Sistema Nervoso Autônomo. Rev Bras Terap Saúde. 2014;5(1):15-20.

7. Kurebayashi LF, Oguisso T, Freitas GF. Acupuntura na enfermagem brasileira: dimensão ético-legal. Acta Paul Enferm. 2009;22(2):210-2.

8. Conselho Federal de Enfermagem (COFEN). Resolução COFEN $n^{\circ}$ 326/2008. Regulamenta no Sistema COFEN/CORENs a atividade de acupuntura e dispõe sobre o registro da especialidade. Brasília (DF):COFEN; 2008. [citado 2017 Out 18]. Disponível em: <http://www. cofen.gov.br/resoluao-cofen-n-3262008_5414.html>.

9. Hoga LA, Reberte LM. Técnicas corporais em Grupo de Gestantes: a experiência dos participantes. Rev Bras Enferm. 2006;59(3):308-13.

10. Spielberger $C D$, Gorsuch RL, Lushene RE. Inventário de ansiedade traço-estado. Tadução de Biaggio AM, Natalício L. Rio de Janeiro: CEPA; 1979.

11. Souza MP. Tratado de auriculoterapia. Brasília (DF): Novo Horizonte; 2012.

12. Reichmann BT. Auriculoterapia - Fundamentos de acupuntura auricular. $3^{\mathrm{a}}$ edição. Curitiba: Estante Virtual; 2002

13. Mascarenhas VH, Lima TR, Silva FM, Negreiros FS, Santos JD, Moura MA, et al. Evidências científicas sobre métodos não farmacológicos para alívio a dor do parto. Acta Paul Enferm. 2019;32(3):350-7.

14. Almeida MS, Nunes MA, Camey S, Pinheiro AP, Schmidt MI. Transtornos mentais em uma amostra de gestantes da rede de atenção básica de saúde no Sul do Brasil. Cad Saude Publica. 2012;28(2):385-93.

15. Araújo DM, Pereira NL, Kac G. Ansiedade na gestação, prematuridade e baido peso ao nascer: uma revisão sistemática da literatura. Cad Saúde Pública.2007;23(3):747-56.

16. Nascimento SR, Amorim MH, Primo CC, Castro DS. Fatores de risco para o desenvolvimento de depressão na gestação. Rev Bras Pesqui Saúde. 2009;11(2):18-9.
17. Buss C, Lord C, Wadiwalla M, Hellhammer DH, Lupien SJ, Meaney MJ, et al. Maternal care modulates the relationship between prenatal risk and hippocampal volume in women but not in men. J Neurosci. 2007 ;27(10):2592-5.

18. Gouveia VV, Chaves SS, Oliveira IC, Dias MR, Gouveia RS, Andrade PR. A utilização do QSG-12 na população geral: estudo de sua validade de construto. Psicol Teor Pesqui. 2003;19(3):241-8.

19. Ferreira LA, Silva JA, Zuffi FB, Mauzalto AC, Leite CP, Nunes JS. Expectativas das gestantes em relação ao parto. Ver Pesqui Cuid Fundam Online 2013;5(2):3692-97.

20. Costa CE, Gotlieb SL. Estudo epidemiológico do peso ao nascer a partir da Declaração de Nascido Vivo. Rev Saude Publica. 1998;32(4):328-34.

21. Horta BL, Barros FC, Halpern R, Victora CG. Baixo peso ao nascer em duas coortes de base populacional no Sul do Brasil. Cad Saude Publica. 1996;12 Suppl 1:S27-31.

22. Amorim MH, Repossi CD, Leite LD. Avaliação do nível de ansiedade e imunoglobulina $A$ em mulheres de parto normal e cesárea. In: $11^{\circ}$ Congresso Panamericano de Profesionales de Enfermería e 55 Congresso Brasileiro de Enfermagem, 2003, Novembro 3-7; Rio de Janeiro, Rio de Janeiro. Rio de Janeiro: ABEn; 2003.

23. Heron J, O'Connor TG, Evans J, Golding J, Glover V; ALSPAC Study Team. The course of anxiety and depression through pregnancy and the postpartum in a community sample. J Affect Disord. 2004;80(1):65-73.

24. Primo C,Amorim M. Efeitos do relaxamento na ansiedade e nos níveis de IgA salivar de puérperas. Rev Lat Am Enfermagem. 2008;16(1):36-41.

25. Benson H. The relaxion response. In: Goleman D, editor. GURIN J. Mind/body medicine- how to use your mind for better health. Boston: Consumers Reports Books; 1993. p. 233-57.

26. Brasil. Ministério da Saúde. Secretaria de Atenção à Saúde. Departamento de Atenção Básica. Manual de implantação de serviços de práticas integrativas e complementares no SUS / Ministério da Saúde, Secretaria de Atenção à Saúde, Departamento de Atenção Básica. - Brasília (DF): Ministério da Saúde, 2018. 